\title{
Wireless MIMO switching: distributed zero-forcing and MMSE relaying using network coding
}

\author{
Miao Wang, Fanggang Wang ${ }^{*}$ and Zhangdui Zhong
}

\begin{abstract}
In a switching problem, a one-to-one mapping from the inputs to the outputs is conducted according to a switch pattern, i.e., a permutation matrix. In this paper, we investigate a wireless switching problem, in which a group of single-antenna relays acts together as a multiple-input-multiple-output (MIMO) switch to carry out distributed precode-and-forward. All users transmit simultaneously to the MIMO switch in the uplink and then the MIMO switch precodes the received signals and broadcasts in the downlink. Ideally, each user could receive its desired signal from one other user with no or little interference from other users. Self-interference is allowed in the received signals, as it can be canceled when each user has the channel gain of its self-interference. We propose two distributed relaying schemes based on two widely adopted criteria, i.e., zero-forcing relaying and minimum mean square error (MMSE) relaying. For the distributed zero-forcing relaying, we further propose a message passing approach, with which the proposed zero-forcing relaying achieves significant throughput gain with little attendant overhead. We also claim that the proposed MMSE relaying achieves even larger throughput at the expense of larger amount of message passing. Simulation results validate the throughput gains of the proposed relaying schemes.
\end{abstract}

\section{Introduction}

Two-way relaying has attracted tremendous attention due to its potential of significantly improving the spectral efficiency [1-4]. By applying physical-layer network coding (PNC) [5], two half-duplex nodes can accomplish a bidirectional data exchange in two phases with the assist of a half-duplex relay.

Recently, much of the interest has been generalized to multi-way relaying [6-17]. The major challenge of designing a multi-way relaying system lies in the co-channel interference induced by multiple users, which could dramatically degrade the system performance if not handled properly. So far, most related works focus on two traffic patterns, i.e., pairwise data exchange where the users form pairs to exchange data within each pair, and full data exchange, where each user and full data exchange, where each user broadcasts to all other users [8-11]. In contrast, the traffic pattern studied in our earlier papers $[12,13,16,17]$ is an arbitrary unicast, where the mapping

\footnotetext{
${ }^{*}$ Correspondence: wangfg@bjtu.edu.cn

State Key Laboratory of Rail Traffic Control and Safety, EIE, Beijing Jiaotong University, Beijing, China
}

from transmitters to receivers can be an arbitrary permutation and thus is more general. Such a framework is called wireless multiple-input-multiple-output (MIMO) switching [12], and it is realized via a multi-antenna relay. In this paper, we follow the same framework and study the relaying schemes of the distributed relays.

Specifically, we investigate distributed relaying schemes for multiple single-antenna relays in the wireless MIMO switching network. Most of the existing works on multiway relaying including our previous works exploited a multi-antenna relay to improve relaying efficiency $[6,7,10$ $13,16,17]$. However, due to limited size and power, etc., it is natural to deploy multiple single-antenna relays in the network. A group of such relays forms a MIMO switch where distributed beamforming is carried out. Since data exchange among the distributed relays is expensive, decode-and-forward schemes which involve joint coding and decoding at the relays are not practical. Thus, non-regenerative relaying is used instead in the network. In addition, the precoding matrix at the relays is diagonal as each relay can only multiply its received

\section{Springer}

(c) 2013 Wang et al: licensee Springer. This is an Open Access article distributed under the terms of the Creative Commons Attribution License (http://creativecommons.org/licenses/by/2.0), which permits unrestricted use, distribution, and reproduction in any medium, provided the original work is properly cited. 
signal with a complex gain. The schemes for a multiple antenna relay fail for distributed relays. In [14], the authors proposed an orthogonalize-and-forward protocol to make multiple pairs of two-way transmissions independent with the aid of multiple single-antenna relays. In this paper, we propose a scheme to improve the system sum rate which chooses a precoding vector in the interference null space under the criterion of maximizing the ratio of overall signal power to noise power. In [15], it was assumed that the users do not have knowledge of the channel state information. In order to achieve interference-free transmission at the users, the equivalent channel gains of each desired signal and its corresponding self-interference signal are designed to be equal to 1 , and the gains of inter-user to 0 with proper precoding at the relays citemolisch11. We will show that by limited attendant message limited attendant message passing, the constraint that the channel gains of desired signals and self-interferences are equal can be relaxed. By doing so, the minimum required number of relays is reduced, we could achieve higher throughput. Furthermore, both citeBor07 and citemolisch11 assume that the [15] assume that the uplink and downlink channels are reciprocal. We will indicate that the relaying schemes in $[14,15]$ fail for nonpairwise traffic pattern and solve this problem later in this paper.

As we know, zero-forcing schemes could boost noise at low signal-to-noise ratio (SNR) or for an ill-conditioned channel. Minimum mean square error (MMSE) schemes strike a balance between interference and noise. MMSE precoder and receiver were investigated in [18] for a single-hop transmission. Similar MMSE schemes with a multi-antenna relay were proposed to minimize sum mean square error (MSE) of all the users in $[7,11]$ for multi-way relaying. In contrast, due to the practical issues aforementioned, we propose a distributed MMSE relaying scheme to circumvent the noise-elevating issue of zero-forcing schemes.

Specifically, in this paper, we first propose a distributed zero-forcing relaying scheme, which nulls all inter-user interferences and maximizes the ratio of overall signal power to noise power. For this relaying scheme, we also propose a message-passing approach with low overhead. After that, we propose a distributed MMSE relaying which minimizes the sum MSE and improves the throughput further by jointly optimizing the precoders at the relays and the receive filters at the users. We aim to deploy both relaying schemes for pairwise/non-pairwise switching under reciprocal/nonreciprocal channels. For nonpairwise switching in reciprocal channels, a desired signal and the interference signal of its reverse link belong to the same signal space, which could make the proposed schemes fail. A trick by using the conjugate of the received signals at each hop is provided to deal with this problem.
Notation. The operators $(\cdot)^{\mathrm{T}},(\cdot)^{*},(\cdot)^{\mathrm{H}}$, and $(\cdot)^{-1}$ denote the transposition, the complex conjugation, the Hermitian and the inverse of a matrix, respectively. $\odot$ denotes the Hadamard product, i.e., element-by-element product. $\operatorname{Tr}\{\mathbf{W}\}$ and $\operatorname{rank}(\mathbf{W})$ denote the trace and the rank of $\mathbf{W}$, respectively. $\mathbb{E}[\cdot]$ is the expectation of a random variable. $\mathbf{W}=\operatorname{diag}\{\mathbf{w}\}$ denotes a diagonal matrix with the elements of $\mathbf{w}$ on the diagonal. $\mathbf{w}=\operatorname{diag}\{\mathbf{W}\}$ denotes a column vector that contains the diagonal elements of the matrix $\mathbf{W}$. $[\mathbf{W}]_{\text {diag }}$ denotes a diagonal matrix with the same diagonal elements as $\mathbf{W}$.

The remainder of this paper is organized as follows. The system setup of a distributed MIMO switching is introduced in Section 2. Two distributed relaying schemes based on zero-forcing and MMSE criteria are proposed in Sections 3 and 4, respectively. In Section 5, special cases of the two relaying schemes are further investigated in reciprocal channels. The simulation results are presented in Section 6. The paper is concluded in Section 7.

\section{System model}

Consider a wireless MIMO switching network with $K$ single-antenna users and $N$ distributed single-antenna relay nodes which form a virtual MIMO switch as shown in Figure 1 . The $K$ users communicate with each other via the MIMO switch, assuming that direct links are not available between any two users. In this paper, we focus on a pure unicast traffic pattern where each user transmits to one other user only. We assume that each user and

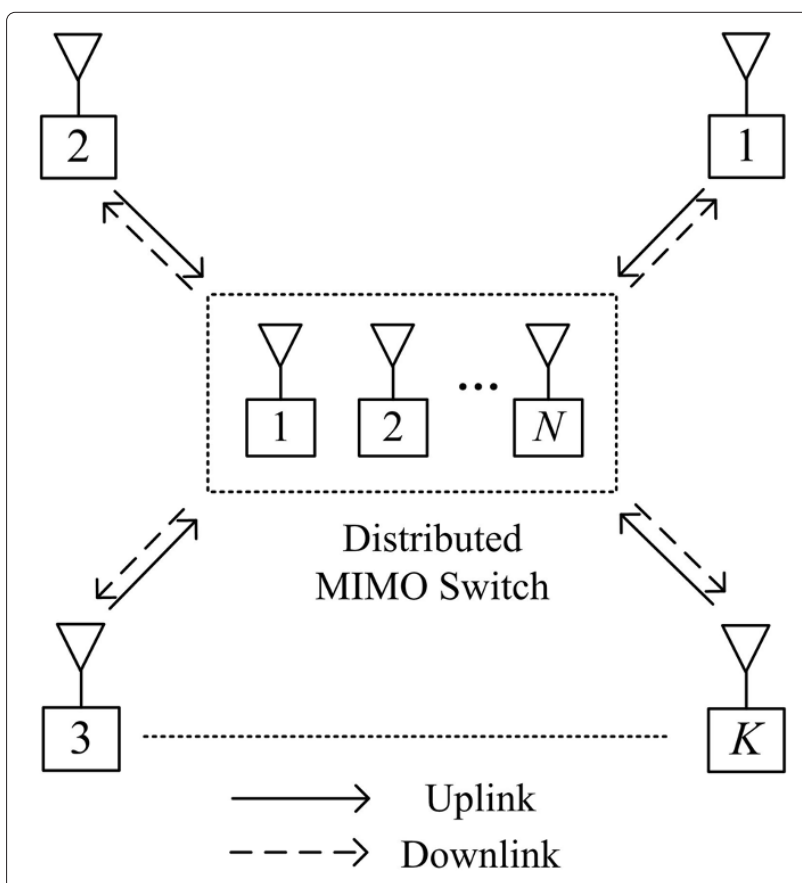

Figure 1 Distributed wireless MIMO switching. 
relay is half-duplex, and then each transmission consists of two phases. In the uplink phase, all users transmit their signals simultaneously to the relays. After that, the relays broadcast their precoded signals back to the users in the downlink phase.

Let $\mathbf{x}=\left[x_{1}, \cdots, x_{K}\right]^{\mathrm{T}}$ be the signal vector of the users satisfying $\mathbb{E}\left[\mathbf{x x}^{\mathrm{H}}\right]=\mathbf{I}_{K}$. The received signals at the relays can be expressed as

$$
\mathbf{y}=\mathbf{H x}+\mathbf{u},
$$

where $\mathbf{H}=\left(\mathbf{h}_{1}, \cdots, \mathbf{h}_{K}\right)$ is the uplink channel matrix, and $\mathbf{h}_{k} \in \mathbb{C}^{N \times 1}$ is the channel vector from user $k$ to the $N$ relays; $\mathbf{u} \in \mathbb{C}^{N \times 1}$ is the noise vector at the relays, with independent and identically distributed (i.i.d.) noise following a circularly symmetric complex Gaussian (CSCG) distribution, i.e., $u_{n} \sim \mathcal{C N}\left(0, \sigma^{2}\right)$. Upon receiving the uplink signal, each relay scales its received signal by a complex gain before forwarding to the users, that is,

$$
\mathbf{z}=\mathbf{G y}
$$

where $\mathbf{G}=\operatorname{diag}\{\mathbf{g}\}$, and the $n$th element of $\mathbf{g}$ is the complex gain factor at relay $n \mathbf{G}$ is designed to realize a particular switch pattern among the users, which is specified by a mapping $\pi(\cdot)$. That is to say, user $i$ sends data to user $\pi(i)$ through the relays in a transmission. The total transmission power of the relays is bounded by $P_{R}$, i.e.,

$$
\begin{aligned}
\mathbb{E}\left\{\|\mathbf{G} \mathbf{y}\|^{2}\right\} & =\operatorname{Tr}\left\{\mathbf{G}\left(\mathbf{H} \mathbf{H}^{\mathrm{H}}+\gamma^{2} \mathbf{I}_{N}\right) \mathbf{G}^{\mathrm{H}}\right\} \\
& =\mathbf{g}^{\mathrm{H}} \Phi \mathbf{g} \leq P_{\mathrm{R}},
\end{aligned}
$$

where $\Phi \triangleq\left[\mathbf{H} \mathbf{H}^{\mathrm{H}}+\gamma^{2} \mathbf{I}_{N}\right]_{\text {diag. }}$. The received signals at the users can be written as

$$
\mathbf{r}=\mathbf{F G H x}+\mathbf{F G u}+\mathbf{w},
$$

where $\mathbf{w} \in \mathbb{C}^{K \times 1}$ is the noise vector at the users, with i.i.d. noise samples following a CSCG distribution, i.e., $w_{k} \sim$ $\mathcal{C N}\left(0, \sigma^{2}\right)$. Equivalently, the received signal of user $\pi(i)$ is explicitly rewritten as

$$
\begin{aligned}
r_{\pi(i)}= & \underbrace{\mathbf{f}_{\pi(i)}^{\mathrm{T}} \mathbf{G h} x_{i}}_{\text {desired signal }}+\underbrace{\mathbf{f}_{\pi(i)}^{\mathrm{T}} \mathbf{G h} \mathbf{h}_{\pi(i)} x_{\pi(i)}}_{\text {self-interference }} \\
& +\underbrace{\sum_{j \neq i, \pi(i)} \mathbf{f}_{\pi(i)}^{\mathrm{T}} \mathbf{G h}_{j} x_{j}}_{\text {inter-user interference }}+\underbrace{\mathbf{f}_{\pi(i)}^{\mathrm{T}} \mathbf{G u}+w_{\pi(i)}}_{\text {noise }},
\end{aligned}
$$

where the downlink channel is written as $\mathbf{F}=$ $\left(\mathbf{f}_{1}, \cdots, \mathbf{f}_{K}\right)^{\mathrm{T}}$, and $\mathbf{f}_{k} \in \mathbb{C}^{N \times 1}$ is the channel vector from the relays to user $k$. The first term of the right hand side of (6) is the desired signal; the second term is the selfinterference which can be canceled by user $\pi(i)$ as long as the equivalent channel gain $\mathbf{f}_{\pi(i)}^{T} \mathbf{G h}_{\pi(i)}$ is known to this user; the third term is the inter-user interference induced by other users; the last two terms are noises from the relays and the user end. Assuming that the upper- and downlink phase both consume half of the total intervals, we obtain the sum rate as

$$
R=\frac{1}{2} \sum_{i=1}^{K} \log _{2}\left(1+\frac{\left|\mathbf{f}_{\pi(i)}^{\mathrm{T}} \mathbf{G} \mathbf{h}_{i}\right|^{2}}{\sum_{j \neq i, \pi(i)}\left|\mathbf{f}_{\pi(i)}^{\mathrm{T}} \mathbf{G h}_{j}\right|^{2}+\sigma^{2}\left|\mathbf{f}_{\pi(i)}^{\mathrm{T}} \mathbf{G}\right|^{2}+\sigma^{2}}\right)
$$

In the following discussion, we optimize the sum rate based on two different criteria in the distributed MIMO switching network.

\section{Distributed zero-forcing relaying}

In this section, we propose a distributed zero-forcing relaying scheme that eliminates all interferences at the users. Specifically, we use a vector in the null space of the total inter-user interferences as the precoder. By properly choosing the vector, we can maximize the ratio of the overall signal power to noise as well. In what follows, we elaborate the details of this scheme.

The total power of inter-user interference at user $\pi(i)$ is written as

$$
\begin{aligned}
P_{\pi(i)}^{\mathrm{int}} & =\mathbb{E}\left[\left(\sum_{j \neq i, \pi(i)} \mathbf{f}_{\pi(i)}^{\mathrm{T}} \mathbf{G h}_{j} x_{j}\right)\left(\sum_{j \neq i, \pi(i)} \mathbf{f}_{\pi(i)}^{\mathrm{T}} \mathbf{G h}_{j} x_{j}\right)^{\mathrm{H}}\right] \\
& =\sum_{j \neq i, \pi(i)} \mathbf{f}_{\pi(i)}^{\mathrm{T}} \mathbf{G h}_{j} \mathbf{h}_{j}^{\mathrm{H}} \mathbf{G}^{\mathrm{H}} \mathbf{f}_{\pi(i)}^{*} \\
& =\sum_{j \neq i, \pi(i)} \mathbf{g}^{\mathrm{T}} \operatorname{diag}\left(\mathbf{f}_{\pi(i)}\right) \mathbf{h}_{j} \mathbf{h}_{j}^{\mathrm{H}} \operatorname{diag}\left(\mathbf{f}_{\pi(i)}^{*}\right) \mathbf{g}^{*} \\
& =\mathbf{g}^{T}\left(\sum_{j \neq i, \pi(i)}\left(\mathbf{f}_{\pi(i)} \odot \mathbf{h}_{j}\right)\left(\mathbf{f}_{\pi(i)} \odot \mathbf{h}_{j}\right)^{\mathrm{H}}\right) \mathbf{g}^{*} .
\end{aligned}
$$

Let

$$
\mathbf{W}_{\pi(i)}=\left(\sum_{j \neq i, \pi(i)}\left(\mathbf{f}_{\pi(i)} \odot \mathbf{h}_{j}\right)\left(\mathbf{f}_{\pi(i)} \odot \mathbf{h}_{j}\right)^{\mathrm{H}}\right)^{*} .
$$

To null the inter-user interference of user $\pi(i)$, the precoder vector $\mathbf{g}$ satisfies

$$
\mathbf{g}^{\mathrm{T}} \mathbf{W}_{\pi(i)}^{*} \mathbf{g}^{*}=0
$$

Then, all inter-user interferences of the users being zero can be equivalently expressed as

$$
\mathbf{g}^{\mathrm{H}} \mathbf{W g}=0 \text {, }
$$

where $\mathbf{W}=\sum_{i=1}^{K} \mathbf{W}_{i}$. Since each user has $K-2$ independent inter-user interferences, the total number of independent inter-user interferences is $K(K-2)$ when the uplink and downlink channels are nonreciprocal. ${ }^{a}$ In this case, the number of relays is required to be larger than $K(K-2)$ 
to guarantee each inter-user interference to be zero, i.e., $N>K(K-2)$.

From (14), it is obvious that $\mathbf{g}$ is in the null space of $\mathbf{W}$. Since $\mathbf{W}$ is the sum of $K(K-2)$ different interference subspaces, the rank of $\mathbf{W}$ is $K(K-2)$ with probability of 1. The orthonormal basis of the null space of $\mathbf{W}$ denoted as $\mathbf{N}_{\mathbf{W}} \in \mathbb{C}^{N \times(N-K(K-2))}$ here can be obtained by the singular value decomposition:

$$
\mathbf{W}=\left[\begin{array}{ll}
\mathbf{S}_{\mathbf{W}} & \mathbf{N}_{\mathbf{W}}
\end{array}\right] \Lambda_{\mathbf{W}} \mathbf{V}_{\mathbf{W}}^{\mathrm{H}} .
$$

The solution to (14) is thus a linear combination of the columns of $\mathbf{N}_{\mathbf{W}}$ and can be written as

$$
\mathbf{g}=\mathbf{N}_{\mathbf{W}} \alpha,
$$

where $\alpha=\left[\alpha_{1}, \cdots, \alpha_{N-K(K-2)}\right]^{T}$ is the linear combination vector. We then determine the weights $\alpha$ by maximizing the ratio of the total power of the desired signals to the noise power. The total desired signal power is written as

$$
\begin{aligned}
P^{\mathrm{sig}} & =\mathbb{E}\left[\left(\sum_{i=1}^{K} \mathbf{f}_{\pi(i)}^{T} \mathbf{G h}_{i} x_{i}\right)\left(\sum_{i=1}^{K} \mathbf{f}_{\pi(i)}^{\mathrm{T}} \mathbf{G h}_{i} x_{i}\right)^{\mathrm{H}}\right] \\
& =\mathbf{g}^{T}\left(\sum_{i=1}^{K}\left(\mathbf{f}_{\pi(i)} \odot \mathbf{h}_{i}\right)\left(\mathbf{f}_{\pi(i)} \odot \mathbf{h}_{i}\right)^{\mathrm{H}}\right) \mathbf{g}^{*} \\
& =\mathbf{g}^{\mathrm{H}}\left(\sum_{i=1}^{K}\left(\mathbf{f}_{\pi(i)} \odot \mathbf{h}_{i}\right)^{*}\left(\mathbf{f}_{\pi(i)} \odot \mathbf{h}_{i}\right)^{\mathrm{T}}\right) \mathbf{g} \\
& =\alpha^{\mathrm{H}} \Omega \alpha
\end{aligned}
$$

where $\Omega=\mathbf{N}_{\mathbf{W}}\left[\sum_{i=1}^{K}\left(\mathbf{f}_{\pi(i)} \odot \mathbf{h}_{i}\right)^{*}\left(\mathbf{f}_{\pi(i)} \odot \mathbf{h}_{i}\right)^{\mathrm{T}}\right] \mathbf{N}_{\mathbf{W}}^{\mathrm{H}} \cdot$ The total noise power is

$$
\begin{aligned}
P^{\text {noise }} & =\mathbb{E}\|\mathbf{F G u}+\mathbf{w}\|^{2} \\
& =\sigma^{2} \operatorname{Tr}\left\{\mathbf{F G} \mathbf{G}^{\mathrm{H}} \mathbf{F}^{\mathrm{H}}+\mathbf{I}\right\} \\
& =\sigma^{2} \mathbf{g}^{\mathrm{H}}\left[\mathbf{F}^{\mathrm{H}} \mathbf{F}\right]_{\operatorname{diag}} \mathbf{g}+K \sigma^{2} \\
& =\sigma^{2} \alpha^{\mathrm{H}} \Gamma \alpha+K \sigma^{2}
\end{aligned}
$$

where $\Gamma=\mathbf{N}_{\mathbf{W}}^{\mathrm{H}}\left[\mathbf{F}^{\mathrm{H}} \mathbf{F}\right]_{\text {diag }} \mathbf{N}_{\mathbf{W}}$. The ratio of the total power of desired signal to the total noise power $\eta$ can be obtained as

$$
\eta=\frac{P^{\text {sig }}}{P^{\text {noise }}}=\frac{\alpha^{\mathrm{H}} \Omega \alpha}{\sigma^{2}\left(\alpha^{\mathrm{H}} \Gamma \alpha+K\right)} .
$$

By inserting (15) into (4), the total power constraint at the relays can be rewritten as

$$
\begin{aligned}
\mathbf{g}^{\mathrm{H}} \Phi \mathbf{g} & =\alpha^{\mathrm{H}} \mathbf{N}_{\mathbf{W}}^{\mathrm{H}} \Phi \mathbf{N}_{\mathbf{W}} \alpha \\
& =\alpha^{\mathrm{H}} \Phi^{\prime} \alpha \leq P_{R},
\end{aligned}
$$

where $\Phi^{\prime}=\mathbf{N}_{\mathbf{W}}^{\mathrm{H}} \Phi \mathbf{N}_{\mathbf{W}}$. We claim that the relays need to transmit with total power $P_{\mathrm{R}}$ to maximize $\eta$. It can be proved as follows: suppose $\alpha_{\text {opt }}$ is the optimal combination vector and $\alpha_{\mathrm{opt}}^{\mathrm{H}} \Phi^{\prime} \alpha_{\mathrm{opt}}<P_{\mathrm{R}}$, we can always find a $\alpha=\rho \alpha_{\mathrm{opt}}$ to make $\alpha^{\mathrm{H}} \Phi^{\prime} \alpha=P_{\mathrm{R}}$ with $\rho>1$. For $\alpha$, the corresponding $\eta_{\alpha}$ is

$$
\begin{aligned}
\eta_{\alpha} & =\frac{\alpha^{\mathrm{H}} \Omega \alpha}{\sigma^{2}\left(\alpha^{\mathrm{H}} \Gamma \alpha+K\right)} \\
& =\frac{\alpha_{\mathrm{opt}}^{\mathrm{H}} \Omega \alpha_{\mathrm{opt}}}{\sigma^{2}\left(\alpha_{\mathrm{opt}}^{\mathrm{H}} \Gamma \alpha_{\mathrm{opt}}+\frac{K}{\rho^{2}}\right)} \\
& >\frac{\alpha_{\mathrm{opt}}^{\mathrm{H}} \Omega \alpha_{\mathrm{opt}}}{\sigma^{2}\left(\alpha_{\mathrm{opt}}^{\mathrm{H}} \Gamma \alpha_{\mathrm{opt}}+K\right)}=\eta_{\alpha_{\mathrm{opt}}},
\end{aligned}
$$

which contradicts with that $\alpha_{\mathrm{opt}}$ is optimal. Thus, the optimal $\alpha$ satisfies the equation $\frac{\alpha^{\mathrm{H}} \Phi^{\prime} \alpha}{P_{\mathrm{R}}}=1$. Denote that $\Gamma^{\prime}=$ $\Gamma+\frac{K}{P_{\mathrm{R}}} \Phi^{\prime}$. We can rewrite $\eta$ as

$$
\eta=\frac{\alpha^{\mathrm{H}} \Omega \alpha}{\sigma^{2} \alpha^{\mathrm{H}} \Gamma^{\prime} \alpha}
$$

which is a generalized Rayleigh ratio problem [19]. The optimal $\alpha$ to maximize $\eta$ is $\alpha=\kappa \bar{\alpha}$ where $\bar{\alpha}$ is the eigenvector of $\left(\Gamma^{\prime}\right)^{-1} \Omega$ corresponding to its largest eigenvalue, and $\kappa$ is a scaling factor to guarantee that the relays transmit with maximum power in (4), i.e.,

$$
\kappa=\sqrt{\frac{P_{\mathrm{R}}}{\bar{\alpha}^{\mathrm{H}} \Phi^{\prime} \bar{\alpha}}} .
$$

Remark 1. To perform self-interference cancelation, the equivalent channel response of self-interference $\mathbf{f}_{\pi(i)}^{T} \mathbf{G h}_{\pi(i)}$ in (6) is needed at user $\pi(i)$. In addition, the equivalent channel response of desired signal $\mathbf{f}_{\pi(i)}^{T} \mathbf{G h}_{i}$ in (6) is also required at user $\pi(i)$ to estimate the desired signal. We claim that the equivalent channel gains can be delivered within at most three steps for an arbitrary unicast pattern. We provide an example to show how the users get the required channel responses. Consider seven users with traffic demands as shown in Figure 2. If only

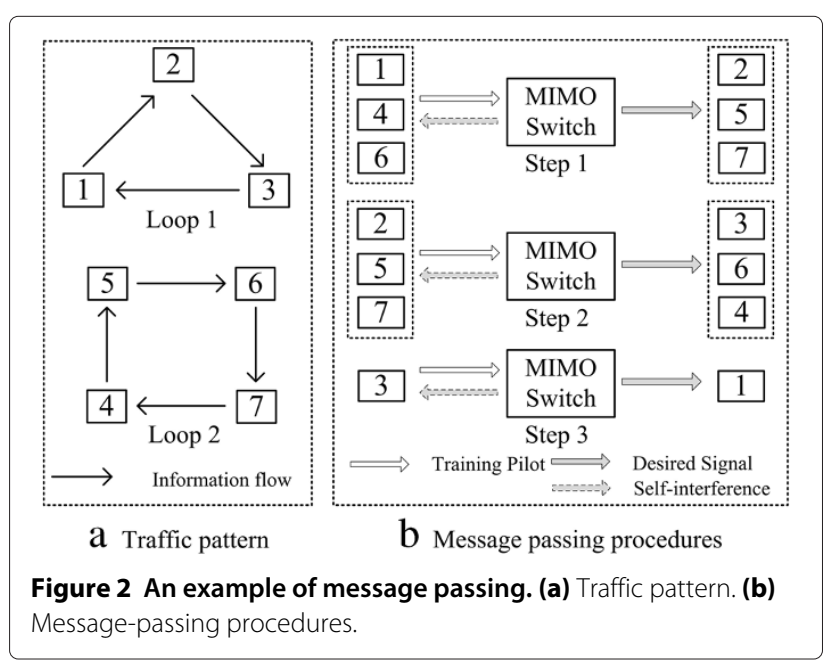


user $i$ (e.g., user 1 ) sends a training pilot to the relays and the relays broadcast the precoded signal back to the users, in such a transmission, user $i$ (e.g., user 1) obtains the gain of its self-interference and user $\pi(i)$ (e.g., user 2 ) gets the channel gain of the desired signal link. Since zero-forcing relaying nulls inter-user interference completely, multiple users could send training pilots simultaneously without interfering each others, as long as users $i, \pi^{-1}(i)$, and $\pi(i)$ (e.g., users 1,3 , and 2) do not transmit together. Based on this requirement, the users in a unicast cycle with even users can be scheduled in two steps, i.e., adjacent users are scheduled in different steps. The users in a unicast cycle with odd users can be scheduled in three steps. Note that the users from different cycles can be scheduled together because different cycles do not interfere with each other. As illustrated in Figure 2, the required channel information needed can be obtained in three steps shown in Figure $2 \mathrm{~b}$ for the switching pattern in Figure 2a. In the first transmission, users 1,4 , and 6 estimate the equivalent channel gains of self-interferences, and users 2,5 , and 7 estimate the gains of the desired signals. In the second transmission, users 2,5 , and 7 estimate the equivalent channel gains of self-interferences, and users 3,4 , and 6 estimate the gains of the desired signals. In the last transmission, user 3 estimates the equivalent channel gain of self-interference, and user 1 estimates the gain of its desired signal. Thus, we conclude that at most three steps are required for message passing before data transmission of the network.

Note that a central node is required to compute the precoding matrix at the relays following the assumption in $[16,17]$. In addition, channel information $\mathbf{H}$ and $\mathbf{F}$ are required at the relays. The channel estimation at the relays is out of the scope of this paper. More details can be found in $[20,21]$.

\section{Distributed MMSE relaying}

In this section, we propose a distributed MMSE relaying scheme that minimizes the sum MSE by jointly optimizing the precoder at each relay and the receive filter at each user end.

Similar to [13], we use a diagonal matrix $\mathbf{B}$ to express the weights of self-interference and a diagonal matrix $\mathbf{C}$ to denote the receive filters. The receivers first filter the signal with $\mathbf{C}$ and then subtract the self-interferences $\mathbf{B x}$ from the filtered signal $\mathbf{C r}$. The sum MSE can be written as $\mathbb{E}\left\{\|\mathbf{P x}-(\mathbf{C r}-\mathbf{B x})\|^{2}\right\}$, where $\mathbf{P}$ is a $K$-dimensional permutation matrix realizing the required switch pattern, i.e., the mapping defined by $\pi(\cdot)$ as mentioned in Section 2; that is, column $i$ of $\mathbf{P}$ is equal to $\mathbf{e}_{\pi(i)}$, i.e., $\mathbf{p}_{i}=\mathbf{e}_{\pi(i)}$, where $\mathbf{e}_{i}$ is column $i$ of an identity matrix. Together with the total transmission power constraint of the relays in (4), the sum MSE minimization problem is formulated as follows:

$$
\begin{array}{rl}
\min _{\mathbf{G}, \mathbf{B}, \mathbf{C}} & \mathbb{E}\left\{\|(\mathbf{P}+\mathbf{B}) \mathbf{x}-\mathbf{C r}\|^{2}\right\} \\
\text { s.t. } & \mathbf{g}^{\mathrm{H}} \Phi \mathbf{g} \leq P_{\mathrm{R}}, \\
& \mathbf{B}, \mathbf{C} \in \mathbb{C}^{K \times K} \text { are diagonal. }
\end{array}
$$

Unfortunately, the optimization problem (33a) is nonconvex and thus is generally difficult to solve in an optimal way. We then propose an iterative algorithm to optimize G, B, and C. A similar method was used in [7,18], which considered a single-hop problem or a multi-way relaying without network coding. Here we show that PNC can be easily incorporated and optimized in the iterative method.

To solve the problem (33), we introduce a cofactor $\beta$ and define

$$
\overline{\mathbf{G}}=\beta^{-1} \mathbf{G} \quad \text { and } \quad \overline{\mathbf{C}}=\beta \mathbf{C} .
$$

The Lagrangian function is written as

$$
\begin{aligned}
\mathcal{L}(\overline{\mathbf{g}}, \mathbf{B}, \overline{\mathbf{C}}, \lambda)= & \mathbb{E}\left\{\|(\mathbf{P}+\mathbf{B}) \mathbf{x}-\mathbf{C r}\|^{2}\right\}+\lambda\left(\mathbf{g}^{\mathrm{H}} \Phi \mathbf{g}-P_{\mathrm{R}}\right) \\
= & \operatorname{Tr}\left\{\mathbf{I}+\mathbf{B} \mathbf{B}^{\mathrm{H}}-2 \Re\left\{\overline{\mathbf{C}} \mathbf{F} \overline{\mathbf{G}} \mathbf{H}(\mathbf{P}+\mathbf{B})^{\mathrm{H}}\right\}\right. \\
& +\overline{\mathbf{C}} \mathbf{F} \overline{\mathbf{G}} \mathbf{H} \mathbf{H}^{\mathrm{H}} \overline{\mathbf{G}}^{\mathrm{H}} \mathbf{F}^{\mathrm{H}} \overline{\mathbf{C}}^{\mathrm{H}} \\
& \left.+\gamma^{2} \overline{\mathbf{C}} \mathbf{F} \overline{\mathbf{G}}^{\mathrm{H}} \overline{\mathbf{F}}^{\mathrm{H}} \overline{\mathbf{C}}^{\mathrm{H}}+\sigma^{2} \beta^{-2} \overline{\mathbf{C}} \overline{\mathbf{C}}^{\mathrm{H}}\right\} \\
& +\lambda\left(\beta^{2} \overline{\mathbf{g}}^{\mathrm{H}} \Phi \overline{\mathbf{g}}-P_{\mathrm{R}}\right),
\end{aligned}
$$

where $\overline{\mathbf{g}}=\operatorname{diag}\{\overline{\mathbf{G}}\} \in \mathbb{C}^{N \times 1}$, and the last step follows by plugging (5) and (34).

\subsection{Optimal $(\bar{g}, \beta)$ for fixed $(B, \bar{C})$}

For fixed $(\mathbf{B}, \overline{\mathbf{C}})$, the Lagrangian function (36) can be rewritten as

$$
\begin{aligned}
\mathcal{L}(\overline{\mathbf{g}}, \lambda)= & \operatorname{Tr}\left\{\mathbf{I}+\mathbf{B B}^{\mathrm{H}}+\sigma^{2} \beta^{-2} \overline{\mathbf{C}} \overline{\mathbf{C}}^{\mathrm{H}}\right\} \\
& -2 \Re\left\{\mathbf{d}^{\mathrm{H}} \overline{\mathbf{g}}\right\}+\overline{\mathbf{g}}^{\mathrm{H}} \mathbf{S} \overline{\mathbf{g}}+\lambda\left(\beta^{2} \overline{\mathbf{g}}^{\mathrm{H}} \Phi \overline{\mathbf{g}}-P_{\mathrm{R}}\right),
\end{aligned}
$$

where

$$
\begin{aligned}
& \mathbf{d}=\operatorname{diag}\left\{\mathbf{F}^{\mathrm{H}} \overline{\mathbf{C}}^{\mathrm{H}}(\mathbf{P}+\mathbf{B}) \mathbf{H}^{\mathrm{H}}\right\} \in \mathbb{C}^{N \times 1}, \\
& \mathbf{S}=\left(\mathbf{F}^{\mathrm{H}} \overline{\mathbf{C}}^{\mathrm{H}} \overline{\mathbf{C}} \mathbf{F}\right) \odot\left(\mathbf{H} \mathbf{H}^{\mathrm{H}}+\gamma^{2} \mathbf{I}_{N}\right)^{T} \in \mathbb{C}^{N \times N} .
\end{aligned}
$$

From the KKT conditions, we have

$$
\begin{aligned}
& \frac{\partial \mathcal{L}(\overline{\mathbf{g}}, \lambda)}{\partial \overline{\mathbf{g}}^{*}}=0 \longrightarrow \overline{\mathbf{g}}=\left(\mathbf{S}+\lambda \beta^{2} \Phi\right)^{-1} \mathbf{d}, \\
& \frac{\partial \mathcal{L}(\overline{\mathbf{g}}, \lambda)}{\partial \lambda}=0 \longrightarrow \beta=\sqrt{\frac{P_{\mathrm{R}}}{\overline{\mathbf{g}}^{\mathrm{H}} \Phi \overline{\mathbf{g}}}} \\
& \frac{\partial \mathcal{L}(\overline{\mathbf{g}}, \lambda)}{\partial \beta}=0 \longrightarrow \lambda \beta^{4} \overline{\mathbf{g}}^{\mathrm{H}} \Phi \overline{\mathbf{g}}=\sigma^{2} \operatorname{Tr}\left\{\overline{\mathbf{C}} \overline{\mathbf{C}}^{\mathrm{H}}\right\} .
\end{aligned}
$$

Substituting (41) into (42), we get

$$
\lambda \beta^{2}=\frac{\sigma^{2} \operatorname{Tr}\left\{\overline{\mathbf{C}} \overline{\mathbf{C}}^{\mathrm{H}}\right\}}{P_{\mathrm{R}}} .
$$


Plugging (43) to (40), we have

$$
\overline{\mathbf{g}}=\left(\mathbf{S}+\frac{\sigma^{2} \operatorname{Tr}\left\{\overline{\mathbf{C}} \overline{\mathbf{C}}^{\mathrm{H}}\right\}}{P_{\mathrm{R}}} \Phi\right)^{-1} \mathbf{d} \text {. }
$$

Note that similar KKT conditions were considered in [7]. However, the derivation provided here is much simpler.

\subsection{Optimal $(\mathrm{B}, \overline{\mathrm{C}})$ for fixed $(\overline{\mathrm{g}}, \boldsymbol{\beta})$}

Denote $\mathbf{b}=\operatorname{diag}\{\mathbf{B}\} \in \mathbb{C}^{K \times 1}, \overline{\mathbf{c}}=\operatorname{diag}\{\overline{\mathbf{C}}\} \in \mathbb{C}^{K \times 1}$. For fixed $(\overline{\mathbf{g}}, \beta)$, i.e., fixed $\mathbf{G}$, the Lagrangian function can be rewritten as

$$
\begin{aligned}
\mathcal{L}(\mathbf{b}, \overline{\mathbf{c}})= & K+\mathbf{b}^{\mathrm{H}} \mathbf{b}-\overline{\mathbf{c}}^{\mathrm{H}} \mathbf{s}-\overline{\mathbf{c}}^{\mathrm{H}} \mathbf{Q}_{1}^{\mathrm{H}} \mathbf{b}-\mathbf{s}^{\mathrm{H}} \overline{\mathbf{c}}-\mathbf{b}^{\mathrm{H}} \mathbf{Q}_{1} \overline{\mathbf{c}} \\
& +\beta^{-2} \overline{\mathbf{c}}^{\mathrm{H}} \mathbf{Q}_{2} \overline{\mathbf{c}}+\lambda\left(\beta^{2} \overline{\mathbf{g}}^{\mathrm{H}} \Phi \overline{\mathbf{g}}-P_{\mathrm{R}}\right) \\
= & K+\left(\mathbf{Q}_{3} \overline{\mathbf{c}}-\mathbf{Q}_{3}^{-1} \mathbf{s}\right)^{\mathrm{H}}\left(\mathbf{Q}_{3} \overline{\mathbf{c}}-\mathbf{Q}_{3}^{-1} \mathbf{s}\right)-\mathbf{s}^{\mathrm{H}} \mathbf{Q}_{3}^{-2} \mathbf{s} \\
& +\left(\mathbf{b}-\mathbf{Q}_{1} \overline{\mathbf{c}}\right)^{\mathrm{H}}\left(\mathbf{b}-\mathbf{Q}_{1} \overline{\mathbf{c}}\right)+\lambda\left(\beta^{2} \overline{\mathbf{g}}^{\mathrm{H}} \Phi \overline{\mathbf{g}}-P_{\mathrm{R}}\right),
\end{aligned}
$$

where

$$
\begin{aligned}
\mathbf{s} & =\operatorname{diag}\left\{\mathbf{P} \mathbf{H}^{\mathrm{H}} \overline{\mathbf{G}}^{\mathrm{H}} \mathbf{F}^{\mathrm{H}}\right\} \in \mathbb{C}^{K \times 1}, \\
\mathbf{Q}_{1} & =[\mathbf{F} \overline{\mathbf{G}} \mathbf{H}]_{\operatorname{diag}} \in \mathbb{C}^{K \times K}, \\
\mathbf{Q}_{2} & =\left[\mathbf{F} \overline{\mathbf{G}}\left(\mathbf{H} \mathbf{H}^{\mathrm{H}}+\gamma^{2} \mathbf{I}\right) \overline{\mathbf{G}}^{\mathrm{H}} \mathbf{F}^{\mathrm{H}}+\sigma^{2} \beta^{-2} \mathbf{I}\right]_{\operatorname{diag}} \in \mathbb{C}^{K \times K}, \\
\mathbf{Q}_{3} & =\left(\mathbf{Q}_{2}-\mathbf{Q}_{1}^{\mathrm{H}} \mathbf{Q}_{1}\right)^{\frac{1}{2}} \in \mathbb{C}^{K \times K} .
\end{aligned}
$$

From (45), we obtain the solution to minimize the sum MSE for fixed $(\overline{\mathbf{g}}, \beta)$,

$$
\begin{aligned}
\mathbf{b} & =\mathbf{Q}_{1} \mathbf{Q}_{3}^{-2} \mathbf{s}, \\
\overline{\mathbf{c}} & =\mathbf{Q}_{3}^{-2} \mathbf{s} .
\end{aligned}
$$

Note that from (51) the interference canceled at the user end is rewritten as

$$
\mathbf{b}=\mathbf{Q}_{1} \mathbf{c}=\operatorname{diag}(\mathbf{C F G H}),
$$

which implies that self-interference is completely canceled by the solution of $\mathbf{B}$ in (51).

By performing the above two steps iteratively, we present the proposed iterative approach as follows:

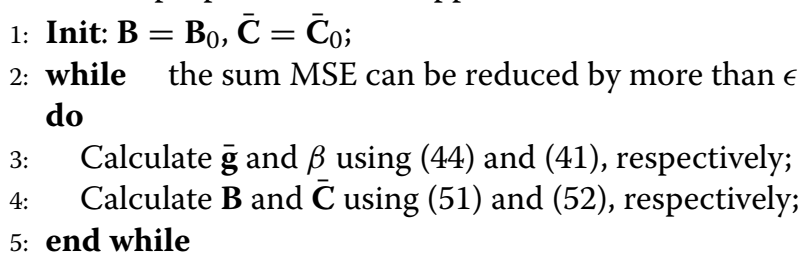

\subsection{Convergence of the iterative algorithm}

The sum MSE decreases monotonically in the iteration. Together with the fact that the sum MSE is nonnegative, we conclude that the iterative algorithm converges. Figure 3 shows the sum rate versus the number of iteration with different numbers of random initial vectors. When

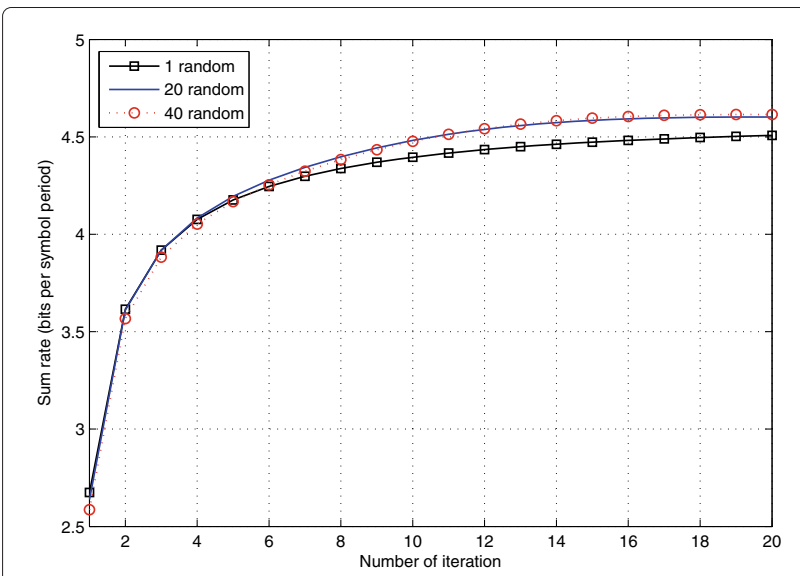

Figure 3 Convergence of the proposed iterative algorithm for MMSE relaying (SNR $\left.=5 \mathrm{~dB}, N=N_{1}, K=4\right)$.

multiple random initial vectors are used, we choose the vector that converges to the highest sum rate. It can be observed that the sum rate converges within 20 iterations. Higher sum rate can be achieved by setting various random initials, but the performance gain is marginal when the number of random initials exceeds 20 .

Remark 2. With the proposed MMSE relaying, each user also demands the equivalent channel gains of the desired signal and the self-interference to recover the desired signal, as that for the proposed zero-forcing relaying mentioned previously in Remark 1 . However, each user needs to send its pilot individually in different steps, since there is residual inter-user interference at the users for the MMSE relaying. Larger overheads are required compared to the proposed zero-forcing scheme. In the simulations, we will show that with the larger overhead, the MMSE relaying can further improve the sum rate that achieved by the proposed zero-forcing scheme.

Remark 3. Here we compare the computational complexity of our schemes with the existing schemes in [14, $15]$. We count the numbers of matrix inversion and singular value decomposition (SVD) needed in each scheme as the complexity of other operations can be neglected compared to them. In the scheme proposed in [14], the major operation is an SVD operation of a $N \times K(K-2)$ interference matrix with complexity $\mathcal{O}\left(N K^{2}(K-2)^{2}\right)$. For the scheme in [15], the major operations are the SVD of a $N \times K(K-2)$ matrix and a matrix inverse operation of two $N \times N$ matrices. The computational complexity is $\mathcal{O}\left(N K^{2}(K-2)^{2}\right)+\mathcal{O}\left(2 N^{3}\right)$. In our proposed zero-forcing scheme, to get the weighting vector (15) at the relays, we need to compute $\mathbf{N}_{\mathbf{W}}$ and $\alpha$. Specifically, we need to compute the null space of a $N \times K(K-2)$ to obtain $\mathrm{N}_{\mathbf{w}}$. To 
get $\alpha$, we need to compute the inverse of a $N \times N$ matrix and the eigenvector of a $N \times N$ matrix. The computational complexity is $\mathcal{O}\left(N K^{2}(K-2)^{2}\right)+\mathcal{O}\left(2 N^{3}\right)$. With MMSE relaying, we need to compute the inverse matrix of a $N \times N$ matrix to obtain $(\overline{\mathbf{g}}, \beta)$ for fixed $(\mathbf{B}, \overline{\mathbf{C}})$ and the matrix inverse of a $K \times K$ matrix to obtain $(\mathbf{B}, \overline{\mathbf{C}})$ for fixed $(\overline{\mathbf{g}}, \beta)$ in each iterative step. Suppose $M$ ( $M$ is less than 20 as evaluated in Figure 3 ) iterations are needed. The computational complexity is $\mathcal{O}\left(M N^{3}\right)+\mathcal{O}\left(M K^{3}\right)$.

\section{Further discussion in reciprocal channels}

We consider the case that the uplink channel and downlink channel are reciprocal, i.e., $\mathbf{F}=\mathbf{H}^{\mathrm{T}}$, which is a widely adopted assumption for time-division duplex systems. In this case, the equivalent channel from user $i$ to user $j$ is exactly the same as that from user $j$ to user $i$. In what follows, we investigate whether the proposed schemes can be used as well in the case of channel reciprocity.

\subsection{Distributed zero-forcing relaying}

We notice that for reciprocal channels, the situations for pairwise and non-pairwise traffic patterns are different as will be elaborated later in this subsection. We first refer to pairwise switching.

\subsubsection{Pairwise switching}

Recall that there are $K(K-2)$ independent inter-user interferences in nonreciprocal channels. Due to channel reciprocity, the interference from user $i$ to user $j, j \neq$ $\pi(i)$ (its equivalent channel is $\mathbf{h}_{j}^{T} \mathbf{G} \mathbf{h}_{i}$ ) and that from user $j$ to user $i$ (its equivalent channel is $\mathbf{h}_{i}^{T} \mathbf{G} \mathbf{h}_{j}$ ) belongs to the same space spanned by the equivalent channel vector $\mathbf{h}_{i} \odot \mathbf{h}_{j}$. With this result, the number of relays required to null all inter-user interferences is larger than $\frac{1}{2} K(K-2)$, which is half of the required number for the nonreciprocal case.

\subsubsection{Non-pairwise switching}

For a non-pairwise switching, if the link from user $i$ to user $j$ is for a desired signal transmission, i.e., $j=\pi(i)$, then the reverse link from user $j$ to user $i$ is an interference link, i.e., $i \neq \pi(j)$ (or else it becomes a pairwise switching). In the case of channel reciprocity, the desired signal and the interference in its reverse link are in the same space spanned by the equivalent channel vector $\mathbf{h}_{i} \odot \mathbf{h}_{j}$. Thus, the interference can not be nulled by projection. We next provide a trick here to differentiate the link from its reverse link.

In the downlink, the relays send the conjugate of the precoded signal $\mathbf{z}$ in (2) instead, i.e.,

$$
\mathbf{z}^{*}=\mathbf{G}^{*} \mathbf{y}^{*}=\mathbf{G}^{*} \mathbf{H}^{*} \mathbf{x}^{*}+\mathbf{G}^{*} \mathbf{u}^{*}
$$

At the user end, all users detect $\mathbf{r}^{\prime}$, which is the conjugate of the received signals, i.e.,

$$
\mathbf{r}^{\prime}=\mathbf{H}^{\mathrm{H}} \mathbf{G H} \mathbf{x}+\mathbf{H}^{\mathrm{H}} \mathbf{G u}+\mathbf{w}^{*} .
$$

With this trick, the equivalent channel gain of the link from user $i$ to user $j$ becomes $\mathbf{h}_{j}^{\mathrm{H}} \mathbf{G} \mathbf{h}_{i}$, and its reverse channel gain is $\mathbf{h}_{i}^{\mathrm{H}} \mathbf{G} \mathbf{h}_{j}$. Since the spaces spanned by $\mathbf{h}_{i} \odot \mathbf{h}_{j}^{*}$ and $\mathbf{h}_{i}^{*} \odot \mathbf{h}_{j}$ are different, the problem becomes the same as the one for the nonreciprocal case and can be solved accordingly.

Remark 4. The minimum relays required in different cases are listed in Table 1 . Recall the schemes in $[14,15]$ that were proposed for multi-user two-way relaying, i.e., pairwise traffic pattern, assuming channel reciprocity. They can be directly extended to the case of channel nonreciprocity, while with different minimum required relays. However, the schemes in $[14,15]$ cannot be extended to non-pairwise switching for reciprocal channels. The proposed conjugate trick could also heal the two proposed schemes.

\subsection{Distributed MMSE relaying}

For the MMSE scheme, the requirement of minimum relays is not necessary. However, for the non-pairwise switching with the assumption of channel reciprocity, even with sufficient relays, it is still difficult to improve the performance because the interferences and the desired signals are coupled together. The throughput can be boosted when the spaces of signal and interference are

Table 1 The minimum relays required for different relaying schemes

\begin{tabular}{lcc}
\hline $\begin{array}{l}\text { Relaying } \\
\text { schemes }\end{array}$ & $\begin{array}{c}\text { Nonreciprocal } \\
\text { Pairwise/non-pairwise }\end{array}$ & Pairwise \\
\hline ZF in [14] & $K(K-2)+1$ & $\frac{1}{2} K(K-2)+1$ \\
ZF in [15] & $K^{2}$ & $\frac{1}{2} K(K+1)$ \\
Proposed ZF & $K(K-2)+1$ & $\frac{1}{2} K(K-2)+1$ \\
Proposed MMSE & & No strict requirement of minimum relays \\
\hline
\end{tabular}


completely decoupled with the trick of using conjugate, which will be shown later in Section 6 .

\section{Numerical results}

In this section, we evaluate the sum MSE and the sum rates of the distributed MIMO switching network using different relaying schemes. Consider a pure unicast traffic pattern with four users, i.e., $K=4$. The maximum total transmission power of the relays is equal to the transmission power of each user, i.e., $P_{\mathrm{R}}=1$. The noise levels at each relay and user are the same. The entries of the channel vectors are generated according to $\mathcal{C N}(0,1)$ and all the results are obtained by averaging 10,000 Monte Carlo simulations. In the simulation, we use $N_{1}$ and $N_{2}$ to denote the required minimum relays for the proposed zero-forcing relaying and the scheme in [15], respectively. Note that the values of $N_{1}$ and $N_{2}$ can be different in different cases as shown in Table 1. Then, we present four observations as follows:

Observation 1. The proposed MMSE relaying scheme has better sum MSE performance than zero-forcing relaying schemes.

In Figure 4, we compare the sum MSE of our proposed schemes with the schemes in $[14,15]$. The simulations show that MMSE relaying outperforms the zero-forcing schemes significantly. With $N_{1}$ relays, i.e., the minimum required relays for the proposed zero-forcing scheme, the scheme in [15] fails with large sum MSE over the whole SNR region. The proposed zero-forcing scheme has the same sum MSE with the scheme in [14] as $\alpha$ is a scalar determined by the power constraint at the relays which is the same for both schemes with $N_{1}$ relays. With MMSE

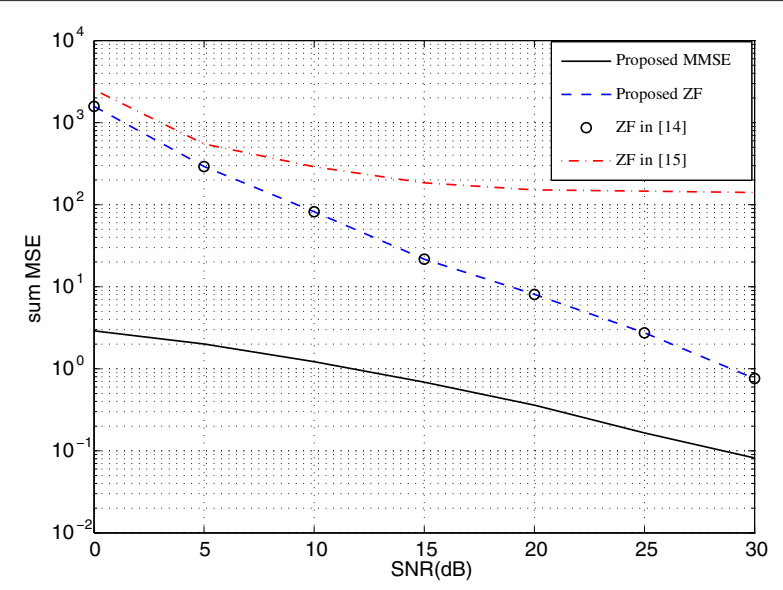

Figure 4 Sum MSE comparison of different relaying schemes $\left(N=N_{1}, K=4\right)$. relaying, sum MSE is reduced significantly as the effect of noise is considered in the design of the scheme.

Observation 2. The proposed distributed zero-forcing and MMSE relaying schemes achieve significant throughput gain over the distributed schemes in $[14,15]$.

In Figures 5, 6, and 7, we compare the sum rates of the two proposed approaches with the relaying schemes in $[14,15]$ in different cases, i.e., Figure 5 for non-pairwise switching with nonreciprocal channels, Figure 6 for pairwise switching with reciprocal channels, and Figure 7 for non-pairwise switching with reciprocal channels. ${ }^{\mathrm{b}}$ It is shown that roughly the same results can be obtained from the three different cases. Both the proposed schemes outperform the schemes in $[14,15]$.

Specifically, from Table 1, we see that the proposed zeroforcing scheme needs less relays than the scheme in [15]. When using $N_{1}$ relays, i.e., the minimum required relays for the proposed zero-forcing scheme, the scheme in [15] fails without efficient throughput. When the number of relays increases to $N_{2}$, i.e., the minimum required relays of the scheme in [15], the proposed schemes still outperform this scheme significantly. Compared to the scheme in [14], the proposed zero-forcing scheme has the same throughput when using $N_{1} y$ relays. This is because with $N_{1}$ relays, $\alpha$ is the same for both schemes as stated above. However, as the number of relays increases, the proposed zeroforcing scheme becomes better. The reason lies in that our scheme always optimizes $\alpha$ to maximize the ratio of overall signal power to noise power. However, the scheme in [16] fails in this.

In all the cases, the proposed MMSE scheme achieves the best throughput performance, and it does not require

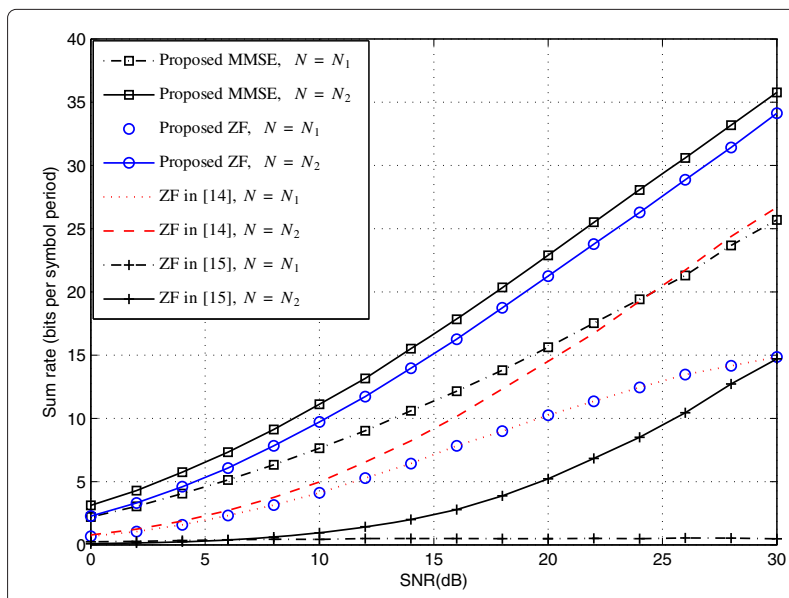

Figure 5 Sum rate comparison of different relaying schemes for non-pairwise switching in nonreciprocal channels $\left(N_{1}=K(K-2)+1, N_{2}=K^{2}, K=4\right)$. 


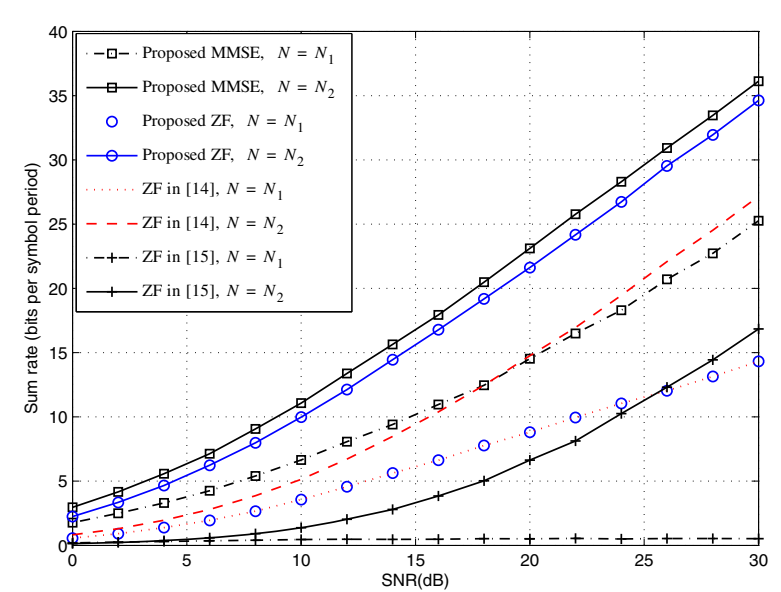

Figure 6 Sum rate comparison of different relaying schemes for pairwise switching in reciprocal channels

$\left(N_{1}=\frac{1}{2} K(K-2)+1, N_{2}=\frac{1}{2} K(K+1), K=4\right)$.

minimum relays. The penalty is that its overhead is larger than the other schemes.

Observation 3. For reciprocal channels, the minimum required relays can be reduced to almost half of the other cases for pairwise switching, and the proposed conjugate trick could avoid the failure of all the schemes for non-pairwise switching.

In Table 1, it can be observed that the number of relays required for pairwise switching in reciprocal channels is almost half of the other cases. The results in Figures 5, 6 , and 7 further indicate that similar sum rate can be achieved in this case with almost half relays.

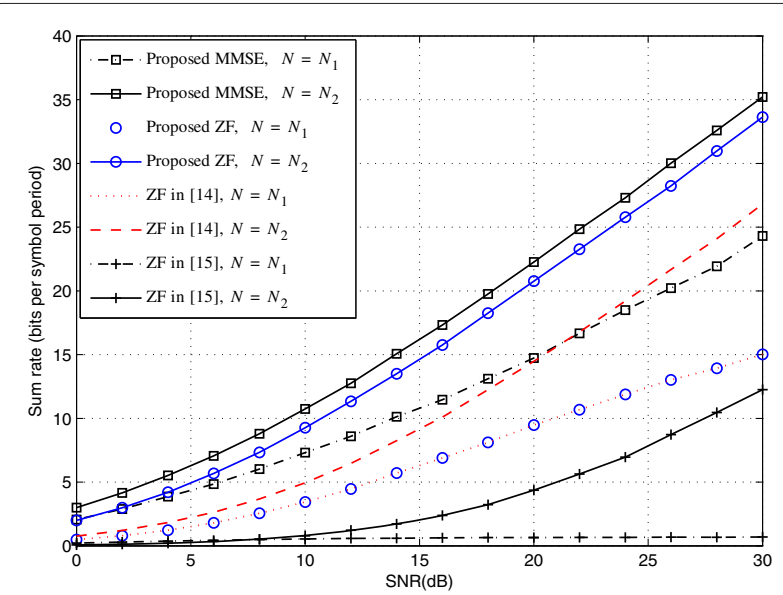

Figure 7 Sum rate comparison of different relaying schemes for non-pairwise switching in reciprocal channels $\left(N_{1}=K(K-2)+1, N_{2}=K^{2}, K=4\right)$.
Figure 8 compares the performance of proposed MMSE relaying with conventional MMSE relaying not using conjugate for non-pairwise switching in reciprocal channels. In this case, the sum rate of conventional MMSE relaying is quite low and can be improved trivially by increasing the number of relays, since the desired signals and some interference signals belong to the same signal space. By using conjugate trick, the problem can be solved and the sum rate increases significantly.

Observation 4. The throughput performance improves as the number of relays increases, specially for the proposed zero-forcing relaying scheme with the number of relays from $N_{1}$ to $N_{1}+1$.

Figure 8 compares the performance of the proposed zero-forcing and MMSE relaying verse different number of relays. We take the non-pairwise switching in reciprocal channels as an example, as similar results can be obtained for other cases. Note that the performance of MMSEbased scheme degrades smoothly when $N$ decreases, which makes this scheme also suitable for smaller system configurations. As for the zero-forcing relaying, one more relay boosts the performance compared to the case with minimum number of relays. However, the throughput improvement is limited when $N$ increases.

\section{Conclusion}

In this paper, two relaying schemes are proposed for the distributed MIMO switching network. We indicate that the proposed distributed zero-forcing scheme exhibits large improvement of system throughput with limited attendant overhead compared to the existing distributed zero-forcing schemes. The proposed distributed

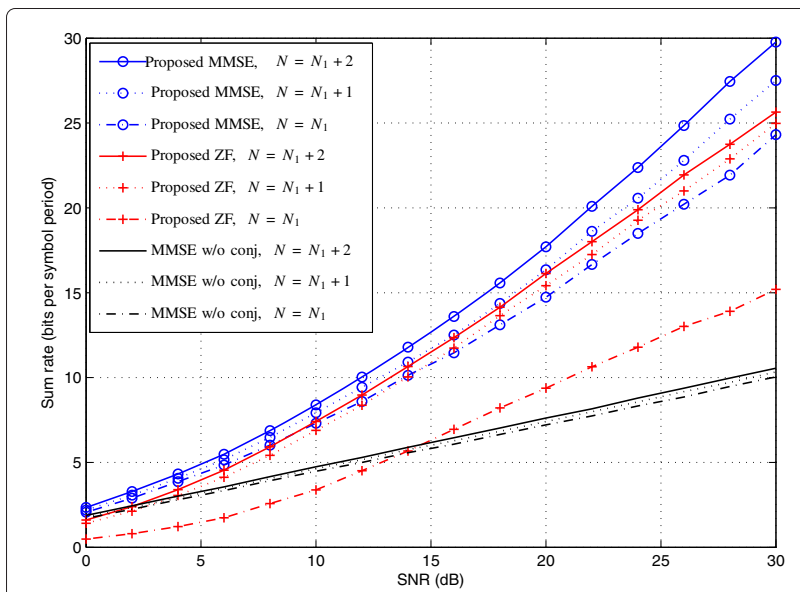

Figure 8 Throughput of proposed zero-forcing and MMSE schemes verse varied $N$ s for non-pairwise switching under reciprocal channels $\left(N_{1}=K(K-2)+1, K=3\right)$. 
MMSE-based scheme can further improve the throughput performance over the proposed zero-forcing scheme at the cost of larger overhead. Both schemes can be applied to an arbitrary unicast pattern under recipro$\mathrm{cal} /$ nonreciprocal channels, with the proposed conjugate trick healing the failure of non-pairwise switching with reciprocal channels.

We assume single-antenna users and relays and perfect channel knowledge at the relays. Future research will focus on the distributed relaying schemes for multiantenna nodes and with partial channel information at the relays.

\section{Endnotes}

a As for reciprocal channels, the number is different, which will be discussed later in Section 5 .

${ }^{b}$ For nonreciprocal channels, the minimum required relays for pairwise and non-pairwise switching is exactly the same. In addition, similar results can be concluded from both pairwise and non-pairwise switching. Thus, we only show the simulation results of non-pairwise switching in Figure 5.

\section{Competing interests}

The authors declare that they have no competing interests.

\section{Acknowledgements}

This work was partially supported by the National Natural Science Foundation (no. 61201201 and U1261109), the State Key Lab of Rail Traffic Control and Safety (no. RCS2011ZT011), the Fundamental Research Funds for the Central Universities (nos. 2011JBM203, 2010RC007, and 2010JBZ008), and the Key Grant Project of Chinese Ministry of education (no. 313006).

Received: 14 June 2012 Accepted: 9 July 2013

Published: 24 July 2013

\section{References}

1. B Rankov, A Wittneben, in Proceedings of the IEEE International Symposium on Information Theory (2006). Achievable rate regions for the two-way relay channel, Seattle, WA, 9-14 July (IEEE, Picataway, 2006), pp. $1668-1672$

2. R Zhang, YC Liang, CC Chai, S Cui, Optimal beamforming for two-way multi-antenna relay channel with analogue network coding. IEEE J. Select. Area Commun. 27(5), 699-712 (2009)

3. R Wang, M Tao, in Proceedings of IEEE International Conference on Commununications (ICC). Joint source and relay precoding designs for MIMO two-way relay systems, Kyoto, 5-9 June (IEEE, Piscataway, 2011), pp. $1-5$

4. T Yang, X Yuan, L Ping, IB Collings, J Yuan, A new physical-layer network coding scheme with eigen-direction alignment precoding for $\mathrm{MIMO}$ two-way relaying. IEEE Trans. Commun. 61(3), 973-986 (2013)

5. S Zhang, SC Liew, PP Lam, in Proceedings of the 12th Annual International Conference on Mobile Computing and Networking. Hot topic: physical-layer network coding, Los Angeles, CA, 24-29 September (ACM, New York, 2006), pp. 358-365

6. Y Mohasseb, H Ghozlan, G Kramer, H El Gamal, in Proceedings of the IEEE International Symposium on Information Theory, The MIMO wireless switch: relaying can increase the multiplexing gain, Seoul, 28 June-3 July (IEEE, Piscataway, 2009), pp. 1448-1552

7. Joung J, Sayed A, Multiuser two-way amplify-and-forward relay processing and power control methods for beamforming systems. IEEE Trans. Signal Proc. 58(3), 1833-1846 (2010)
8. D Gunduz, A Yener, A Goldsmith, HV Poor, in Proceedings of the IEEE International Symposium on Information Theory,. The multi-way relay channel, Seoul, 28 June-3 July (IEEE, Piscataway, 2009), pp. 339-343

9. YE Sagduyu, D Guo, RA Berry, Throughput and stability for relay-assisted wireless broadcast with network coding. IEEE J. Select. Area Commun. 99, $1-11(2012)$

10. TCUi, THo, J Kliewer, in Proceedings of the IEEE Global Telecommunications Conference. Space-time communication protocols for N-way relay networks, San Diego, November (IEEE, Piscataway, 2008), pp. 1-5

11. AUT Amah, A Klein, Beamforming-based physical layer network coding for non-regenerative multi-way relaying. EURASIP J. Wireless Commun. Netw. 2010(12) (2010)

12. F Wang, SC Liew, D Guo, in 49th Annual Allerton Conference on Commununications, Control, and Computing (Allerton). Wireless MIMO switching with zero-forcing relaying, Monticello, IL, 28-30 September (2011), pp. 551-558

13. F Wang, SC Liew, D Guo, Wireless MIMO switching with zero-forcing relaying and network-coded relaying. IEEE J. Select. Area Commun. 30(8), 1452-1463 (2012)

14. B Rankov, A Wittneben, Spectral efficient protocols for half-duplex fading relay channels. IEEE J. Select. Area Comm. 25(2), 379-389 (2007)

15. C Wang, $\mathrm{H}$ Chen, Q Yin, A Feng, AF Molisch, Multi-user two-way relay networks with distributed beamforming. IEEE Trans. Wireless Commun. 10(10), 3460-3471 (2011)

16. F Wang, X Yuan, SC Liew, D Guo, Wireless MIMO switching: weighted sum mean square error and sum rate optimization. IEEE Trans. Inform. Theory (2013). doi:10.1109/TIT.2013.2259893

17. F Wang, Wireless MIMO switching: network-coded MMSE relaying and user group selection. Trans. Emerging Telecommun. Tech. (2012). DOl10.1002/ett.2585

18. M Joham, W Utschick, J Nossek, Linear transmit processing in MIMO communications systems. IEEE Trans. Signal Proc. 53(8), 2700-2712 (2005)

19. HV Trees, L Harry, Detection, Estimation, and Modulation Theory, Part IV: Optimum Array Processing. (Wiley, Hoboken, 2002)

20. T Unger, Multiple-antenna two-hop relaying for bi-directional transmission in wireless communication systems, PhD Thesis, TU Darmstadt, 2009

21. H Degenhardt, F Hohmann, A Klein, in Proceedings of IEEE International Symposium on Personal Indoor and Mobile Radio Commununications (PIMRC). Pilot transmission scheme and robust filter design for non-regenerative multi-pair two-way relaying, Sydney, 9-12 September (IEEE, Piscataway, 2012), pp. 2101-2106

doi:10.1186/1687-6180-2013-130

Cite this article as: Wang et al: Wireless MIMO switching: distributed zero-forcing and MMSE relaying using network coding. EURASIP Journal on Advances in Signal Processing 2013 2013:130.

\section{Submit your manuscript to a SpringerOpen ${ }^{\circ}$ journal and benefit from:}

- Convenient online submission

- Rigorous peer review

- Immediate publication on acceptance

- Open access: articles freely available online

- High visibility within the field

- Retaining the copyright to your article

Submit your next manuscript at springeropen.com 